\title{
CODIMENSION TWO COMPLETE NONCOMPACT SUBMANIFOLDS WITH NONNEGATIVE CURVATURE
}

\author{
MARIA HELENA NORONHA
}

\begin{abstract}
We study the topology of complete noncompact manifolds with nonnegative sectional curvatures isometrically immersed in Euclidean spaces with codimension two. We investigate some conditions which imply that such a manifold is a topological product of a soul by a Euclidean space and this gives a complete topological description of this manifold.
\end{abstract}

\section{INTRODUCTION}

In [9], Sacksteder studied isometric immersions of manifolds with nonnegative sectional curvatures in Euclidean spaces with codimension one, under nondegeneracy conditions about the curvature, namely, that at least one sectional curvature is positive at each point on the manifold. Under the same hypotheses, we want to obtain a topological characterization of complete noncompact manifolds isometrically immersed in codimension two. This uses the existence of a compact soul in $M$, proved by Cheeger and Gromoll in [6]. Baldin and Noronha in [4], show some results along the same line. Basically, it is proved that if this manifold $M^{n}$ is simply connected then $M$ is diffeomorphic to $A^{k} \times \mathbf{R}^{n-k}$, where $A$ is a $k$-dimensional soul of $M$. We obtain a similar conclusion without the simply connected condition and this allows us to know the topology of the manifolds, as we know the topology of the compact soul by [ 2 and 3]. Our first result states

Theorem 1. Let $f: M^{n} \rightarrow \mathbf{R}^{n+2}$ be a substantial isometric immersion of a complete noncompact manifold with nonnegative sectional curvatures, such that at least one of them is positive at each point $x$ in $M$ and let $A^{k}$ be a $k$-dimensional soul of $M$. Then if $k \geq 2, M$ is diffeomorphic to $A^{k} \times \mathbf{R}^{n-k}$ or $\pi_{1}(M)$ is finite. In the latter case $M$ has the homotopy type of the real projective space $\mathbf{R} P^{2}$ or $k=3$.

Remark. In the former case the possibilities for $A^{k}$ follow from [2 and 3]. They are that $A^{k}$ is homeomorphic to a sphere, or a product of two spheres,

Received by the editors April 10, 1987 and, in revised form, December 9, 1987.

1980 Mathematics Subject Classification (1985 Revision). Primary 53C40, 53C42.

Key words and phrases. Compact soul, reducibility along the soul, curvature operator.

The author is partially supported by Fundação de Amparo a Pesquisa do Estado de São Paulo, Brasil. 
or a product of the circle $S^{1}$ by a homotopy sphere, or is diffeomorphic to the total space of a nonorientable fiber bundle uver $S^{1}$ whose fibers are homotopy spheres.

This theorem is proved by showing that the two-dimensional flat torus and also the two-dimensional flat Klein bottle cannot be a soul for this manifold and when $k \geq 3$ we prove that, if $\pi_{1}(M)=\mathbf{Z}$, then the immersion is reducible along a soul $A$ (see Definition (2.7) below). This means that $f$ reduces codimension when restricted to the soul.

Theorem 2. Let $f: M^{n} \rightarrow \mathbf{R}^{n+2}$ be an isometric immersion with the same hypothesis of Theorem 1. If $M$ is simply connected there exists an isometric immersion of the soul $A$ in Euclidean space with codimension two.

This, together with Proposition 3.3 in [2], implies that the complex projective space $\mathbf{C} P^{2}$ cannot be a soul for this manifold $M$.

Before we state our next result, we want to recall that the curvature tensor $R$ at $x$ in $M$ can be regarded as an endomorphism $\mathfrak{R}$ of $T_{x} M \wedge T_{x} M$ which is symmetric with respect to the inner product defined by the Riemannian metric. The hypotheses of the above theorems imply that for each point $x$ in $M$, there exist vectors $U, V$ in $T_{x} M$ such that $\mathfrak{R}(U \wedge V) \neq 0$. A two-form $\mathfrak{R}(U \wedge V)$ is defined to have rank $2 p$ iff $p$ is the largest integer such that $\mathfrak{R}(U \wedge V) \wedge \cdots \wedge \mathfrak{R}(U \wedge V)$ ( $p$ times $) \neq 0$. Since we are studying codimension two, the two-form $\mathfrak{R}(U \wedge V)$ has rank at most 4.

Theorem 3. Let $f: M^{n} \rightarrow \mathbf{R}^{n+2}$ be a substantial isometric immersion of a complete noncompact manifold with nonnegative sectional curvatures and such that for every point $x$ in $M$ there are vectors $U, V$ in $T_{x} M$ such that $\Re(U \wedge V)$ has rank 4. Let $A^{k}$ be a $k$-dimensional soul of $M, k \neq 0$. Then $k \geq 2$ and $M$ is diffeomorphic to $A^{k} \times \mathbf{R}^{n-k}$.

Moreover,

(i) If $k \geq 3$, then $M$ is simply connected.

(ii) If $k=2, A$ is either the sphere $S^{2}$ or the real projective space $\mathbf{R} P^{2}$.

Finally, we will consider the index of relative nullity of $f$ at a point $x$ in $M$ as

$$
\nu_{f}(x)=\operatorname{dim}\left\{X \in T_{x} M: \alpha(X, Y)=0, \forall Y \in T_{x} M\right\}
$$

where $\alpha$ is the second fundamental form. By Hartman [7], if $M$ is not a cylinder, there exists a point $x$ in $M$ such that $\nu_{f}(x)=0$. If this point belongs to a soul we conclude

Theorem 4. Let $f: M^{n} \rightarrow \mathbf{R}^{n+2}$ be a substantial isometric immersion of a complete noncompact manifold with nonnegative sectional curvatures and $k$-dimensional soul $A$. If there is a point $x \in A$ such that $\nu_{f}(x)=0$ we have:

(i) If $k \geq 3, M^{n}$ is simply connected and diffeomorphic to $A^{k} \times \mathbf{R}^{n-k}$, where $A^{k}$ is homeomorphic to the sphere $S^{k}$. 
(ii) If $k=2$ then $M^{n}$ is diffeomorphic to $S^{2} \times \mathbf{R}^{n-2}$ or has the homotopy type of $\mathbf{R} P^{2}$.

We want to observe that the results of Cheeger and Gromoll in [6] do not allow us to know the dimension of the soul. However, under our hypotheses, if the manifold has $\pi_{1}(M)=\mathbf{Z}$ and is not a topological product of a compact manifold by a Euclidean space, we can conclude that the soul is homeomorphic to the circle $S^{1}$.

\section{SOME KNOWN RESULTS OF THE SOUL}

It is a well-known result of Weinstein [10], that if the codimension of an isometric immersion is two then the nonnegativity of the sectional curvatures $(K \geq 0)$ implies the nonnegativity of the curvature operator $(\Re \geq 0)$.

For the case where $M^{n}$ is complete noncompact manifold with $\mathfrak{R} \geq 0$, we now collect some properties of a soul $A$ of $M$. We denote by $A^{k}$ a $k$ dimensional soul of $M, 0 \leq k<n$. We consider the splitting of the tangent bundle of $M, T M=T A \oplus T A^{\perp}$, where $T A$ is the tangent bundle of $A$ and $T A^{\perp}$ is the normal bundle of the inclusion $A \subset M$. We observe the following properties of a soul:

(2.1) If the soul is a point, then $M$ is diffeomorphic to $\mathbf{R}^{n}$. (See [6].)

(2.2) A soul $A$ of $M$ is a compact, totally convex submanifold of $M$ without boundary and has $\mathfrak{R} \geq 0$. (See [6].)

(2.3) The inclusion $i: A \rightarrow M$ is a homotopy equivalence and $M$ is diffeomorphic to the total space of $T A^{\perp}$. (See [6].)

(2.4) If $X \in T A$ and $Y \in T A^{\perp}$, then $\mathfrak{R}(X \wedge Y)=0$. Moreover, $\mathfrak{R}\left(\bigwedge^{2}(T A)\right)$ $\subset \Lambda^{2}(T A)$ and $\mathfrak{R}\left(\bigwedge^{2}\left(T A^{\perp}\right)\right) \subset \Lambda^{2}\left(T A^{\perp}\right)$. (See [3, Lemma 3.1].)

(2.5) The normal curvature tensor $R^{\perp}$ of the inclusion $i: A \rightarrow M$ vanishes. (See [3, Lemma 3.1].)

From these properties we can state the following theorem (proved in [3]).

(2.6) Theorem. If $\pi_{1}(M)=\{0\}$ and $\mathfrak{R} \geq 0$ then $M$ is a topological product of a soul by a Euclidean space.

In order to prove Theorems 1 and 3 in the case that $\pi_{1}(M) \neq\{0\}$, we need an extrinsic property of the immersion, namely, reducibilitiy along the soul.

(2.7) Definition. Let $f: M^{n} \rightarrow \mathbf{R}^{n+p}, p \geq 1$, be an isometric immersion of a complete, noncompact manifold $M$ with $K \geq 0$, nontrivial $k$-dimensional soul $A$ and second fundamental form $\alpha$. We say that $f$ is reducible along $A$ if for $X \in T A$ and $Y \in T A^{\perp}, \alpha(X, Y)=0$.

(2.8) Theorem. If $f$ is reducible along a soul $A$ then $M$ is diffeomorphic to $A^{k} \times \mathbf{R}^{n-k}$. (See [3, Proposition (5.4)].) 
In the rest of this paper $\langle\rangle,, \nabla$ will denote the Riemannian metric and connection respectively. If $\xi$ is a normal direction, $A_{\xi}$ will denote the Weingarten operator and $\nabla^{\perp} \xi$ will be the normal connection.

\section{Basic lemmas. Proof of Theorem 1}

Consider $x \in \dot{A}$. We want to investigate if $f$ satisfies the reducibility condition at $x$. By abuse of notation, we will say " $f$ is reducible at $x$." If for every $X \in T_{x} A, \alpha(X, X)=0$ or for every $Y \in T_{x} A^{\perp}, \alpha(Y, Y)=0$, by the Gauss equation, $f$ is reducible at $x$, since $k \geq 0$ and $\mathfrak{R}(X \wedge Y)=0$.

To study the general case, let $r(x)$ be the Lie algebra generated by the range of the curvature operator $\Re$ at the point $x$. If $U$ is the orthogonal complement of the relative nullity subspace $N(x)$, by Theorem 1 in [5] we have the following possibilities for $r(x)$ :

(a) $r(x)=\bigwedge^{2}(U)$,

(b) $r(x)=\bigwedge^{2}(V) \oplus \bigwedge^{2}(W)$, where $V \oplus W=U$,

(c) $r(x)=u(2)$, the unitary algebra of some complex structure on $U$, if $\operatorname{dim} U=4$.

Moreover, if (b) occurs with $\operatorname{dim} V>1$ and $\operatorname{dim} W>1$ then $V$ and $W$ are orthogonal to each other and $R^{\perp}(x)=0$, where $R^{\perp}$ is the normal curvature tensor of $f$.

(3.2) Lemma. If $\mathfrak{R} \mid \bigwedge^{2}\left(T_{x} A\right) \neq 0$ and $\mathfrak{R} \mid \bigwedge^{2}\left(T_{x} A^{\perp}\right) \neq 0$ then $f$ is reducible at $x$. Moreover, there is an orthonormal frame $\left\{\xi_{1}, \xi_{2}\right\}$ such that $A_{\xi_{1}} \mid T_{x} A=0$ and $A_{\xi_{2}} \mid T_{x} A^{\perp}=0$.

Proof. By (2.4), the only possibility is $r(x)=\Lambda^{2}(V) \oplus \Lambda^{2}(W)$ with $\operatorname{dim} V>1$ and $\operatorname{dim} W>1$ whence $R^{\perp}(x)=0$. Therefore, the lemma follows by Theorem D of [4].

(3.3) Lemma. (a) Suppose $\mathfrak{R} \mid \wedge^{2}\left(T_{x} A\right) \neq 0$ and $\mathfrak{R} \mid \wedge^{2}\left(T_{x} A^{\perp}\right)=0$. If $\alpha(Y, Y)$ $\neq 0$ for some $Y \in T_{x} A^{\perp}$, there is an orthonormal frame $\left\{\xi_{1}, \xi_{2}\right\}$ in the normal space such that rank $A_{\xi_{1}}=1$ and $A_{\xi_{2} \mid T X A^{\perp}}=0$.

(b) If $\mathfrak{R} \mid \bigwedge^{2}\left(T_{x} A\right)=0$ and $\mathfrak{R} \mid \bigwedge^{2}\left(T_{x} A^{\perp}\right) \neq 0$ with $\alpha(X, X) \neq 0$ for some $X \in T_{x} A$, we have a similar conclusion with $A_{\xi_{2}} \mid T_{x} A=0$.

Proof. (a) Consider an orthonormal frame $\left\{X_{1}, \ldots, X_{n}\right\}$ of $T_{x} M$ such that $X_{1}, \ldots, X_{s} \in N(x)$ and $Y \in \operatorname{Span}\left\{X_{1}, \ldots, X_{s}, X_{s+1}\right\}$. We have

$$
\mathfrak{R}\left(X_{i} \wedge X_{j}\right)=0, \quad i=1, \ldots, s \text { and } j=1, \ldots, n .
$$

Denoting by $X^{\prime}$ and $X^{\prime \prime}$ the orthogonal projection of the vector $X$ onto $T_{x} A$ and $T_{X} A^{\perp}$ respectively, by $(2.4)$ we have

$$
\mathfrak{R}\left(Y \wedge X_{j}\right)=\mathfrak{R}\left(Y \wedge X_{j}^{\prime}\right)+\mathfrak{R}\left(Y \wedge X_{j}^{\prime \prime}\right)=0, \quad j=1, \ldots, n .
$$


Then the range of $\Re$ is contained in $\bigwedge^{2}(W)$, where $W=\operatorname{Span}\left\{X_{s+2}, \ldots, X_{n}\right\}$, which implies

$$
r(x) \subset o(n-s-1)
$$

where $o(n-s-1)$ is the orthogonal group. If $n-s=4, r(x)$ cannot be $u(2)$, since $u(2)$ is not contained in $o(3)$. Then $r(x)=\Lambda^{2}(V) \oplus \Lambda^{2}(w)$, where $\operatorname{dim} V=1$.

Then, following the proof of Theorem 1 in [5], there is one normal vector $\xi_{1}$ such that rank $A_{\xi_{1}}=1$. If $\xi_{2}$ is a normal vector orthogonal to $\xi_{1}$ we have

$$
\Re=A_{\xi_{2}} \wedge A_{\xi_{2}} \text {. }
$$

We will prove that this basis $\left\{\xi_{1}, \xi_{2}\right\}$ satisfies the lemma. Consider $X, Y \in$ $T_{x} M$. We have

where

$$
\begin{aligned}
\mathfrak{R}(X \wedge Y)= & \left(A_{\xi_{2}} X\right)^{\prime} \wedge\left(A_{\xi_{2}} Y\right)^{\prime}+\left(A_{\xi_{2}} X\right)^{\prime} \wedge\left(A_{\xi_{2}} Y\right)^{\prime \prime} \\
& +\left(A_{\xi_{2}} X\right)^{\prime \prime} \wedge\left(A_{\xi_{2}} Y\right)^{\prime}+\left(A_{\xi_{2}} X\right)^{\prime \prime} \wedge\left(A_{\xi_{2}} Y\right)^{\prime \prime}
\end{aligned}
$$

$$
\begin{gathered}
\Omega=\left(A_{\xi_{2}} X\right)^{\prime \prime} \wedge\left(A_{\xi_{2}} Y\right)^{\prime \prime}=0, \\
\omega=\left(A_{\xi_{2}} X\right)^{\prime} \wedge\left(A_{\xi_{2}} Y\right)^{\prime \prime}+\left(A_{\xi_{2}} X\right)^{\prime \prime} \wedge\left(A_{\xi_{2}} Y\right)^{\prime}=0
\end{gathered}
$$

since we are supposing $\mathfrak{R} \mid \wedge^{2}\left(T_{x} A^{\perp}\right)=0$. Let us suppose $\left(A_{\xi_{2}} X\right)^{\prime} \neq 0$. Taking interior product of $\omega$ with $\left(A_{\xi_{2}} X\right)^{\prime}$ we get

$$
0=i\left(\left(A_{\xi_{2}} X\right)^{\prime}\right) \omega=\left\|\left(A_{\xi_{2}} X\right)^{\prime}\right\|^{2}\left(A_{\xi_{2}} Y\right)^{\prime \prime}-\left\langle\left(A_{\xi_{2}} Y\right)^{\prime},\left(A_{\xi_{2}} X\right)^{\prime}\right\rangle\left(A_{\xi_{2}} X\right)^{\prime \prime}
$$

and therefore

$$
\left(A_{\xi_{2}} Y\right)^{\prime \prime}=\left\langle\left(A_{\xi_{2}} Y\right)^{\prime},\left(A_{\xi_{2}} X\right)^{\prime}\right\rangle\left\|\left(A_{\xi_{2}} X\right)^{\prime}\right\|^{-2}\left(A_{\xi_{2}} X\right)^{\prime \prime} .
$$

Taking interior product with $\left(A_{\xi_{2}} Y\right)^{\prime}$ we get

$$
\begin{aligned}
0 & =i\left(\left(A_{\xi_{2}} Y\right)^{\prime}\right) \omega=\left\langle\left(A_{\xi_{2}} X\right)^{\prime},\left(A_{\xi_{2}} Y\right)^{\prime}\right\rangle\left(A_{\xi_{2}} Y\right)^{\prime \prime}-\left\|\left(A_{\xi_{2}} Y\right)^{\prime \prime}\right\|^{2}\left(A_{\xi_{2}} X\right)^{\prime \prime} \\
& =\left\|\left(A_{\xi_{2}} X\right)^{\prime}\right\|^{-2}\left\{\left\langle\left(A_{\xi_{2}} Y\right)^{\prime},\left(A_{\xi_{2}} X\right)^{\prime}\right\rangle^{2}-\left\|\left(A_{\xi_{2}} X\right)^{\prime}\right\|^{2}\left\|\left(A_{\xi_{2}} Y\right)^{\prime}\right\|^{2}\right\}\left(A_{\xi_{2}} X\right)^{\prime \prime} .
\end{aligned}
$$

If $\left(A_{\xi_{2}} X\right)^{\prime \prime} \neq 0$ the above relation implies $\left(A_{\xi_{2}} Y\right)^{\prime}=\lambda\left(A_{\xi_{2}} X\right)^{\prime}$ and then

$$
\mathfrak{R}(X \wedge Y)=\left(A_{\xi_{2}} X\right)^{\prime} \wedge\left(A_{\xi_{2}} Y\right)^{\prime}=0 .
$$

Hence,

$$
\text { if } \mathfrak{R}(X \wedge Y) \neq 0 \text { we have }\left(A_{\xi_{2}} X\right)^{\prime \prime}=\left(A_{\xi_{2}} Y\right)^{\prime \prime}=0 .
$$

Consider now the orthonormal basis $\left\{Z_{1}, \ldots, Z_{n}\right\}$ which diagonalizes the operator $A_{\xi_{1}}$ such that $A_{\xi_{1}}\left(Z_{1}\right)=\lambda Z_{1}$ and $A_{\xi_{1}}\left(Z_{i}\right)=0, i \geq 2$. Since $\mathfrak{R} \neq 0$ at $x$, there exist $Z_{i}, Z_{j}$ such that $\mathfrak{R}\left(Z_{i} \wedge Z_{j}\right) \neq 0$. By (3.6), for every $Y \in$ $T_{x} A^{\perp}$ we have $\left\langle\alpha\left(Z_{i}, Y\right), \xi_{2}\right\rangle=\left\langle\alpha\left(Z_{j}, Y\right), \xi_{2}\right\rangle=0$. This implies $\alpha\left(Z_{i}, Y\right)=$ 0 , as we can suppose that $A_{\xi_{1}}\left(Z_{i}\right)=0$. In the Gauss equation this implies 
$\left\langle\alpha\left(Z_{i}, Z_{i}\right), \alpha(Y, Y)\right\rangle=0$, since $\mathfrak{R}\left(Y \wedge Z_{i}\right)=\mathfrak{R}\left(Y \wedge Z_{i}^{\prime}\right)+\mathfrak{R}\left(Y \wedge Z_{i}^{\prime \prime}\right)=0$. Because $\alpha\left(Z_{i}, Z_{i}\right)$ is orthogonal to $\xi_{1}$, we have that $\alpha(Y, Y)$ is orthogonal to $\xi_{2}$. Now, writing the Gauss equation for the sectional curvature of a plane spanned by $X \in T_{x} A$ and $Y \in T_{x} A^{\perp}$, we get

$$
0=\left\langle A_{\xi_{2}} X, X\right\rangle\left\langle A_{\xi_{2}} Y, Y\right\rangle-\left\langle A_{\xi_{2}} Y, X\right\rangle^{2}=-\left\langle A_{\xi_{2}} Y, X\right\rangle^{2} .
$$

This and (3.6) together imply $A_{\xi_{2} \mid T x A^{\perp}}=0$, concluding the proof of (a).

(b) This is proved in an analogous manner.

We observe that, under the hypotheses of Lemma (3.3), in (a) there is only one vector $Y \in T_{x} A^{\perp}$ such that $\alpha(Y, Y) \neq 0$ and in (b) only one vector $X \in T_{x} A$ such that $\alpha(X, X) \neq 0$.

(3.7) Proposition. If $\operatorname{dim} A=k \geq 3$ and $\pi_{1}(M)=\mathbf{Z}$, then $f$ is reducible along $A$.

Proof. Let $\bar{f}=f_{\mid A}: A \rightarrow \mathbf{R}^{n+2}$, the isometric immersion $f$ restricted to the soul. Since $A$ is a totally geodesic submanifold of $M$, the first normal space of $\bar{f}$ is at most two dimensional. We can easily generalize to $\bar{f}$, using the same arguments, Theorems (2.2) and (2.3) of [2], obtaining the same results, since they need only the fact of the first normal space be at most two dimensional. We will denote by $\nu_{f}(x)$ the index of relative nullity of the immersion $\bar{f}$.

Since $A$ is compact, consider $x \in A$ such that $\nu_{\bar{f}}(x)=0$. We claim that $\alpha(Y, Y)=0$, for every $Y \in T_{x} A^{\perp}$. Otherwise, under the conditions of Lemma (3.2), all the sectional curvatures along planes tangent to $A$ at $x$ would be positive. Also, under the conditions of Lemma (3.3), the index of relative nullity would be a $n-k-1$. Then in (3.4) we would have $r(x)=o(k)$. This implies that all the eigenvalues of $A_{\xi_{2}} \mid T_{x} A$ are nonnull and then all the sectional curvatures along planes tangent to $A$ at $x$ would be positive. The slight generalization of Theorem (2.2) of [2] to this immersion $\bar{f}$ would imply that $A$ and consequently $M$, is simply connected.

Now, we will prove the reducibility for $x \in A$ such that $\nu_{\bar{f}}(x)>0$. Let $N_{\gamma}(A)$ denote the set of points in $A$ at which the index of relative nullity is $\gamma$. Since we know that $f$ is reducible on the closure of $N_{0}(A)$, we will use the inductive argument used by Moore to prove Theorem 2 in [8]. Let $\gamma \geq 1$ and $V$ be the open set

$$
N_{\gamma}(A)-\mathrm{Cl}\left[\bigcup\left\{N_{\beta}(A) / \beta<\gamma\right)\right]
$$

where $\mathrm{Cl}$ denotes closure, a set on which the index of relative nullity is equal to the constant $\gamma$.

We recall that if $\pi_{1}(A)=\mathbf{Z}$ by the generalization of Theorem (2.3) of [2], $x$ has a neighborhood isometric to an open subset of the product of the circle $S^{1}$ by a $(k-1)$-dimensional homotopy sphere, which implies that there are 
two integrable and parallel distributions $T_{1}$ and $T_{2}$ such that $\operatorname{dim} T_{1}=1$ and $\operatorname{dim} T_{2}=k-1$.

Suppose that $\mathfrak{R} \mid \bigwedge^{2}\left(T_{x} A\right) \neq 0$ at $x$ and $Z$ is tangent to $T_{1}, Z$ must be relative nullity vector. Otherwise $r(x)=o(m-1) \oplus o(1)$ where $m=k-\gamma$. But in the proof of the Lemma (3.3) we see that $m+1=n-s$, which contradicts (3.4).

Now, consider $\sigma:(a, b) \rightarrow V$ a unit speed geodesic passing through $x$ whose tangent vector $\sigma^{\prime}(t)$ is the relative nullity vector $Z \in T_{1}$, for each $t \in(a, b)$. Assume that $\sigma$ cannot be extended beyond the interval $(a, b)$ without leaving $V$. Since $A$ is compact, either $a>-\infty$ or $b<+\infty$. Suppose $b<+\infty$. By Theorem (6.2) in [1], $\sigma(b)$ lies in the closure of $\bigcup\left\{N_{\beta}(A) / \beta<\gamma\right\}$, a set on which $f$ is reducible by the inductive hypothesis.

We will prove that if $f$ is not reducible at $x, f$ cannot be reducible at $\sigma(b)$, which will be a contradiction. If $f$ is not reducible at $x$ we can take the frame $\left\{\xi_{1}, \xi_{2}\right\}$ of the Lemma (3.3) such that $A_{\xi_{2}} \mid T_{x} A^{\perp}=0$. Let us denote by $X$ and $Y$ the unitary orthogonal projection of $Z_{1}$ (see the proof of Lemma (3.3)) onto $T_{x} A$ and $T_{x} A^{\perp}$ respectively. Denoting by $V$ a vector in $T_{x} A$ orthogonal to $X$ and by $\nabla^{\perp}$ the normal connection, we can apply the Codazzi equation to $X, Y, V$ and $\xi_{2}$ to get

$$
\begin{aligned}
& \left\langle\nabla_{X}^{\perp} \alpha(V, Y), \xi_{2}\right\rangle-\left\langle\alpha\left(\nabla_{X} V, Y\right), \xi_{2}\right\rangle-\left\langle\alpha\left(V, \nabla_{X} Y\right), \xi_{2}\right\rangle \\
& \quad=\left\langle\nabla_{V}^{\perp} \alpha(X, Y), \xi_{2}\right\rangle-\left\langle\alpha\left(\nabla_{V} X, Y\right), \xi_{2}\right\rangle-\left\langle\alpha\left(X, \nabla_{V} Y\right), \xi_{2}\right\rangle .
\end{aligned}
$$

Observe that $\alpha(V, Y)=0$ since $V \in T_{x} A$, is orthogonal to $X$ and $Z_{1}$, $Y \in T_{x} A^{\perp}$ and $\left\langle\alpha(V, Y), \xi_{2}\right\rangle=0$. This together with $A_{\xi_{2}} \mid T_{x} A^{\perp}=0$ and $\nabla_{X} Y \in T_{x} A^{\perp}$ implies that the left-hand side is equal to zero. The same reasons will imply that the right-hand side is equal to

$$
\left\langle\nabla_{V}^{\perp} \alpha(X, Y), \xi_{2}\right\rangle=-\left\langle\alpha(X, Y), \nabla_{V}^{\perp} \xi_{2}\right\rangle=0 .
$$

As we are supposing that $\left\langle\alpha(X, Y), \xi_{1}\right\rangle \neq 0$, we have

$$
\nabla_{V}^{\perp} \xi_{2}=\nabla_{V}^{\perp} \xi_{1}=0
$$

Now, with the same notation we will consider the vector fields $X, Y$ and $Z$ such that on $\sigma(t), Z(t)=\sigma^{\prime}(t)$. Applying the Codazzi equation to $X, Y, Z$ and $\xi_{1}$ we get

$$
\begin{aligned}
& \left\langle\nabla_{X}^{\perp} \alpha(Z, Y), \xi_{1}\right\rangle-\left\langle\alpha\left(\nabla_{X} Z, Y\right), \xi_{1}\right\rangle-\left\langle\alpha\left(Z, \nabla_{X} Y\right), \xi_{1}\right\rangle \\
& \quad=\left\langle\nabla_{Z}^{\perp} \alpha(X, Y), \xi_{1}\right\rangle-\left\langle\alpha\left(\nabla_{Z} X, Y\right), \xi_{1}\right\rangle-\left\langle\alpha\left(X, \nabla_{Z} Y\right), \xi_{1}\right\rangle .
\end{aligned}
$$

As we have observed before, $Z$ must be relative nullity vector and then $Z$ is orthogonal to $X$. This implies in the above equation, that the only nonnull term on the left-hand side is

$$
\begin{aligned}
\left\langle\alpha\left(\nabla_{X} Z, Y\right), \xi_{1}\right\rangle & =\left\langle\nabla_{X} Z, X\right\rangle\left\langle\alpha(X, Y), \xi_{1}\right\rangle+\left\langle\nabla_{X} Z, Y\right\rangle\left\langle\alpha(Y, Y), \xi_{1}\right\rangle \\
& =-\left\langle Z, \nabla_{X} X\right\rangle\left\langle\alpha(X, Y), \xi_{1}\right\rangle
\end{aligned}
$$


since $A$ is totally geodesic. The same reasons will imply that the only nonnull term on the right-hand side is the first one. Therefore the above Codazzi equation is reduced to

$$
\left\langle Z, \nabla_{X} X\right\rangle\left\langle\alpha(X, Y), \xi_{1}\right\rangle=Z\left(\left\langle\alpha(X, Y), \xi_{1}\right\rangle\right)-\left\langle\alpha(X, Y), \nabla_{Z}^{\perp} \xi_{1}\right\rangle .
$$

Since $X \in T_{2}, Z \in T_{1}$, and $T_{2}$ is parallel we have $\left\langle Z, \nabla_{X} X\right\rangle=0$ and by (3.8) we have in (3.9), $Z\left(\left\langle\alpha(X, Y), \xi_{1}\right\rangle\right)=0$. This implies that $\left\langle\alpha(X, Y), \xi_{1}\right\rangle$ is constant on $\sigma(t)$ and then $\left\langle\alpha(X, Y), \xi_{1}\right\rangle \neq 0$ at $\sigma(b)$, which is the required contradiction.

Suppose now that $\mathfrak{R} \mid \bigwedge^{2}\left(T_{x} A\right)=0$ in some neighborhood of $x$. By our hypothesis there is a plane $\sigma$ on $T_{x} M$ such that $k(\sigma)>0$. From Lemma (3.3) we have $\xi_{2}$ such that $A_{\xi_{2}}=0$ and then $\left\langle R_{f}^{\perp}(X, Z) \xi_{1}, \xi_{2}\right\rangle=\left\langle R^{\perp}(X, Z) \xi_{1}, \xi_{2}\right\rangle=0$ for every $X, Z \in T_{x} A$. This implies reducibility at $x$, concluding the proof of the proposition.

In order to prove Theorem 1, first we observe that if the soul $A$ is homeomorphic to the two-dimensional flat torus or flat Klein bottle, as $\mathfrak{R}(X \wedge Z)=0$ for every $X, Z \in T_{x} A$ and every $x \in A$, we would have in Lemma (3.3) $A_{\xi_{2}} \mid T_{x} A=0$ and this would imply $\nu_{\bar{f}}(x)>0$, for each $x \in A$. This is impossible, since $A$ is compact.

Now, Theorem 1 follows from (2.6), (2.8), (3.7) and the generalization of Theorem (2.2) in [2]. The possibilities for $A^{k}$ follow from [2 and 3].

\section{Proof of Theorems 2, 3 and 4}

(4.1) Proof of Theorem 2. Let us consider the normal bundle of $M$ along $A$, denoted by $\left.\nu M\right|_{A}$, and define a normal connection $\widetilde{\nabla}^{\perp}: \mathfrak{X}(M) \times \Gamma\left(\left.\nu M\right|_{A}\right) \rightarrow$ $\Gamma\left(\left.\nu M\right|_{A}\right)$ by $\tilde{\nabla}_{X}^{\perp} \xi=0$ orthogonal projection of $\nabla_{X}^{\perp} \xi$ onto $\left.\nu M\right|_{A}$. Now, as the inclusion of $A$ in $M$ is totally geodesic, we define a second fundamental form $\tilde{\alpha}:\left.T A \oplus T A \rightarrow \nu M\right|_{A}$ by $\tilde{\alpha}(X, Y)=\alpha(X, Y)$. It is clear that $\left.\nu M\right|_{A}, \tilde{\nabla}^{\perp}$ and $\tilde{\alpha}$ verify the Gauss and Codazzi equations. Thus, we need to prove that the condition $\mathfrak{R}(x) \neq 0$ for all $x \in A$, implies the Ricci equation. Denoting by $\bar{\nabla}^{\perp}$ the normal connection for $\bar{f}=\left.f\right|_{A}: A \rightarrow \mathbf{R}^{n+2}$ we have

$$
\nabla_{X}^{\perp} \xi=\nabla_{X}^{\perp} \xi-\sum_{i=n-k+1}^{n}\left\langle\alpha\left(X, Z_{i}\right), \xi\right\rangle Z_{i}
$$

where $\left\{Z_{n-k+1}, \ldots, Z_{n}\right\}$ is an orthonormal frame of $T_{x} A^{\perp}$. Thus, the Ricci equation for $\bar{f}$ is

$$
\begin{aligned}
\left\langle\bar{R}^{\perp}(X, Y) \xi, \eta\right\rangle & =\left\langle R^{\perp}(X, Y) \xi, \eta\right\rangle-\sum_{i=n-k+1}^{n}\left\langle\alpha\left(X, Z_{i}\right), \xi\right\rangle\left\langle\alpha\left(Y, Z_{i}\right), \eta\right\rangle \\
& =\left\langle\left[\tilde{A}_{\xi}, \tilde{A}_{\eta}\right] X, Y\right\rangle
\end{aligned}
$$

where $\tilde{A}_{\xi}: T_{x} A \rightarrow T_{x} A$ is given by $\tilde{A}_{\xi}(X)=$ orthogonal projection of $A_{\xi} X$ onto $T_{x} A$. Since by our definition of $\widetilde{\nabla}_{X}^{\perp} \xi,\left\langle\widetilde{R}^{\perp}(X, Y) \xi, \eta\right\rangle=\left\langle R^{\perp}(X, Y) \xi, \eta\right\rangle$, 
all we need is to prove that

$$
\sum_{i=n-k+1}^{n}\left\langle\alpha\left(X, Z_{i}\right), \xi\right\rangle\left\langle\alpha\left(Y, Z_{i}\right), \eta\right\rangle=0 .
$$

But this follows directly from Lemmas (3.2) and (3.3).

This proves that the soul can be locally isometrically immersed in $\mathbf{R}^{k+2}$. Since $A$ is simply connected, Theorem 2 follows.

(4.2) Proof of Theorem 3. Consider $x \in A$. If $f$ is not reducible at $x$, we will have by Lemma (3.3) a normal vector $\xi_{1}$ such that $\operatorname{rank} A_{\xi_{1}}=1$. Then by the Gauss equation, $\mathfrak{R}(U \wedge V)$ cannot have rank 4 . So $f$ is reducible at $x$.

Now we consider $x$ such that $\nu_{f}(x)=0$. If $\alpha(X, Y)=0$ for every $Y \in$ $T_{x} A^{\perp}$, consider $U, V \in T_{x} A$ such that $\mathfrak{R}(U \wedge V)$ has rank 4. Denoting by $U^{\prime}$ and $U^{\prime \prime}$ the orthogonal projection onto $T_{x} A$ and $T_{x} A^{\perp}$ respectively, we have $\mathfrak{R}(U \wedge V)=\mathfrak{R}\left(U^{\prime} \wedge V^{\prime}\right)$. This implies $k \geq 4$. We claim that $M$ is simply connected. For otherwise, by the generalizations of Theorems (2.2) and (2.3) of [2], we would have $r(x)=o(k-1) \oplus o(1)$ and then there would be a normal vector $\xi$ such that $\operatorname{rank} A_{\xi}=1$, which contradicts $\mathfrak{R}(U \wedge V) \wedge \mathfrak{R}(U \wedge V) \neq 0$.

If there is $Y \in T_{x} A^{\perp}$ such that $\alpha(Y, Y) \neq 0$, we have the conditions of Lemma (3.2) and we can take that normal frame $\left\{\xi_{1}, \xi_{2}\right\}$. Then $k \geq 2$ (otherwise rank $A_{\xi_{1}}$ would be one), and all the sectional curvatures along planes tangent to $A$ are positive. If $k \geq 3, M$ is simply connected. If $k=2, A$ cannot be homeomorphic to flat torus or to the flat Klein Bottle.

Now Theorem (2.8) finishes the proof.

(4.3) Proof of Theorem 4. Consider $x \in M$ such that $\nu_{f}(x)=0$. By (2.4) and (3.1) we have $r(x)=\bigwedge^{2}(V) \oplus \bigwedge^{2}(W)$ where $V \oplus W=T_{x} M$. If $\operatorname{dim} V>1$ and $\operatorname{dim} W>1$ we are under the conditions of Lemma (3.2). If $\operatorname{dim} V=1$ or $\operatorname{dim} W=1$ we are under conditions of Lemma (3.3). In both cases, we have that all the sectional curvatures along planes tangent to $A$ are positive at this point $x$. Again, if $k \geq 3, M$ is simply connected and $A^{k}$ homeomorphic to $S^{k}$. If $k=2$ then $A$ is homeomorphic to $S^{2}$ or $\mathbf{R} P^{2}$. Now, applying Theorem (2.6) to the simply connected case we finish the theorem.

\section{REFERENCES}

1. S. B. Alexander, Reducibility of euclidean immersions of low codimension, J. Differential Geometry 3 (1969), 69-82.

2. Y. Y. Baldin and F. Mercuri, Isometric immersions in codimension two with non-negative curvature, Math. Z. 173 (1980), 111-117.

3. Codimension two nonorientable submanifolds with nonnegative curvature. Proc. Amer. Math. Soc. 103 (1988), 918-920.

4. Y. Y. Baldin and M. H. Noronha, Some complete manifolds with non-negative curvature operator, Math. Z. 195 (1987), 383-390. 
5. R. L. Bishop, The holonomy algebra of immersed manifolds of codimension two, J. Differential Geometry 2 (1968), 347-353.

6. J. Cheeger and D. Gromoll, On the structure of complete open manifolds of non-negative curvature, Ann. of Math. (2) 96 (1972), 413-443.

7. P. Hartman, On the isometric immersions in euclidean space of manifolds with non-negative sectional curvatures. II, Trans. Amer. Math. Soc. 147 (1970), 529-540.

8. J. D. Moore, Isometric immersions of Riemannian products, J. Differential Geometry 5 (1971), 159-168.

9. R. Sacksteder, On hypersurfaces with non-negative sectional curvatures, Amer. J. Math. 82 (1960), 609-630.

10. A. Weinstein, Positively curved n-manifolds in $\mathbf{R}^{n+2}$, J. Differential Geometry 4 (1970), 1-4.

Universidade Estadual de Campinas, IMECC, C. P. 6065, 13081 Campinas, São Paulo, BRASIL 